Hydroécol. Appl. (1996) Tome 8 Vol. 1-2, pp. 173-191

\title{
Flux de matières en suspension et de cadmium dans le Lot
}

\section{Fluxes of suspended matter and cadmium in the Lot river (France)}

Y. Lapaquellerie(1), N. Maillet(1), J.-M. Jouanneau
J. P. Coakley,
(2), C. Latouche

(1) Université de Bordeaux I. Département de Géologie of Océanographie, CNRS URA 197, avenue des Facultés, 33405, Talence, France.

(2) National Water Research Institute, 867 Lakeshore Road, Burlington, Ontario, Canada L7R4A6.

Résumé. - La contamination en cadmium, observée dans les huîtres de l'estuaire de la Gironde, provient, principalement par l'intermédiaire du Lot, d'une ancienne usine de traitement du minerai de zinc et des métaux qui lui sont associés. Les flux de cadmium principalement expulsé sous la forme particulaire sont suivis annuellement depuis 1990 . Un des facteurs repose sur la stratégie et la méthodologie d'échantillonnage. L'important échantillonnage recueilli au niveau des turbidités a permis non seulement d'établir et de comparer deux méthodes de calcul des flux de MES mais aussi de montrer l'importance du régime hydrologique de la rivière en particulier du rôle des crues quant à la remobilisation des sédiments de fonds. Le bilan suffisamment précis des flux de MES a permis d'établir les flux de cadmium particulaire émis avec une approximation suffisante pour permettre de juger des efforts de réhabilitation du site pollueur.

Mots clés. - Flux de MES, Pollution de cadmium particulaire, Flux de cadmium dissous, Processus fluviaux.

Abstract. - The source of present-day cadmium contamination in the Gironde estuary is a now-abandoned industrial facility used for refining zinc ore, located in the upper reaches of the Lot river basin. In quantifying the fluxes of cadmium contamination in the river, a considerable effort had to be devoted to methodological development. A key factor in the methodological scheme was the measurement of fluxes of total suspended particulate matter (TSS) for various regimes during the year. The large set of measurements of turbidity and volumetric discharge at monitoring sites, allowed quantitative comparison of two techniques of calculating TSS fluxes. Using these techniques, values for particulate cadmium flux were calculated which ranged from 1.3 in 1990 (the driest year) to 17.5 t. $y^{-1}$ in 1992 (wet year). Estimation of total cadmium fluxes contributed by the Lot River to such a level of precision are deemed critical in directing clean-up efforts in the basin and estuary as a whole.

Key words. - TSS measurement, Cadmium pollution, Fluvial process, Flux of particulate $\mathrm{Cd}$, Flux of dissolved Cd. 


\section{INTRODUCTION}

La contamination en cadmium des huîtres se développant naturellement à l'embouchure de la Gironde est un fait bien connu (Boutier, 1981). L'origine fluviatile et les processus estuariens aboutissant à cette pollution ont été identifiés à la faveur de diverses recherches sur le comportement des métaux dans l'estuaire de la Gironde (Jouanneau et al., 1990). Dans le cadre d'une étude de l'ensemble des bassins versants de la Dordogne et de la Garonne (Latouche, 1970; Latouche et al., 1989) il a été montré que le cadmium arrivant en Gironde provenait principalement $190 \%$ en 1987) de la région industrielle de Decazeville dans le bassin du Lot (fig. 1), par l'intermédiaire d'un petit affluent, le Riou Mort; celui-ci récupère les eaux de ruissellement provenant des terrils non stabilisés et non isolés ainsi que des rejets de l'Usine de Vieille Montagne. Cette usine s'est installée sur le bassin de Decazeville à Viviez dans l'Aveyron, il y a plus d'un siècle. Une pollution métallique chronique du Lot était connue depuis 1971 (Roux et Simonet, 1987). Les premières interventions visant à réduire les émissions de zinc et de cadmium se sont traduites par le traitement des rejets provenant de l'atelier d'électrolyse pour la production du zinc. Ainsi de 1974 à 1985 les flux de cadmium en provenance de l'usine étaient estimés de 50 à $100 \mathrm{~kg}$ par jour. Après une importante pollution accidentelle en cadmium en juillet 1986 (Boisseau, 1987), des aménagements ont été faits pour isoler les terrils de la nappe phréatique et des ruisseaux et pour réduire au maximum les rejets directs de l'usine. Ils ont permis de limiter de manière très importante les flux de la source polluante qui sont passés d'après les estimations de l'usine de $40 \mathrm{~kg} . j^{-1}$ en 1986, à $1 \mathrm{~kg} . \mathrm{j}^{-1}$ en 1991. En dépit de cette réduction, les sédiments contaminés qui se sont accumulés pendant de nombreuses années dans le lit du Lot sont susceptibles d'entretenir des flux encore élevés de cadmium par remise en suspension des sédiments ou par diffusion au fond. Les contrôles effectués entre 1990 et 1995 sur les eaux et les sédiments du Lot sur 2 stations situées à quelques $\mathrm{km}$ en amont (Boisse Penchot) et quelques $\mathrm{km}$ en aval (Bouillac) de la confluence du Riou Mort indiquent des valeurs faibles à Boisse Penchot 10,85 à $5,15 \mathrm{mg} \cdot \mathrm{kg}^{-1}$ pour les MES et 28 à $70 \mathrm{ng}^{.-1}$ dans les eaux) et des valeurs très fortes à Bouillac (80 à $400 \mathrm{mg}^{\mathrm{kg}} \mathrm{kg}^{-1}$ pour les MES et 500 à 1600 ng. $^{\prime}{ }^{\prime}$ dans les eaux). Ces valeurs sont à comparer à celles citées par Boutier (1990) pour les ordres de grandeur des concentrations en cadmium dans les principaux fleuves français (25 à $200 \mathrm{ng}^{-1} \mathrm{l}^{-1}$ pour le cadmium dissous; 1,5 à $30 \mathrm{mg}^{\mathrm{kg}} \mathrm{g}^{-1}$ pour le cadmium particulaire).

Afin de cerner l'importance de ces flux contaminants, d'en suivre l'évolution et éventuellement de gérer l'avenir de cette situation, une étude précise des flux de cadmium expulsés par le Lot vers la Garonne puis dans l'estuaire de la Gironde a été entre- 
prise sous l'égide de l'Agence de l'Eau Adour-Garonne à partir de 1990. Ces objectifs supposaient un suivi à long terme et faisaient appel à une étude méthodologique visant en particulier à établir un protocole d'échantillonnage rigoureux permettant de parfaire la continuité des études.

Les difficultés principales rencontrées dans cette démarche sont celles relatives à l'établissement d'un bilan des flux de matières en suspension (MES). La précision de ce bilan est importante car les MES sont les principaux vecteurs du cadmium et de divers autres métaux, le zinc en particulier (Jouanneau, 1983). En même temps, une stratégie d'échantillonnage compatible avec le budget de l'étude devait être recherchée. L'élaboration de cette méthodologie, son amélioration progressive, puis les résultats obtenus et leur interprétation constituent l'objet de la communication présentée.

\section{MÉTHODOLOGIE}

\subsection{Echantillonnage}

La mesure des flux de MES et de métaux véhiculés par un fleuve implique la connaissance des paramètres suivants :

- la représentativité du site du prélèvement;

- les débits liquides (variations hydrologiques);

- la charge en MES;

- les concentrations en métaux dissous et particulaire.
La méthodologie utilisée pour caractériser chacun de ces paramètres, ainsi que les modes de calcul retenus pour l'estimation des flux sont successivement exposés ci-dessous.

La stratégie de prélèvement, compte tenu des variations spécifiques de chacun des paramètres de flux, nous a appris qu'il n'était pas nécessaire de faire tous les prélèvements correspondant avec la même fréquence. Les MES étant le facteur le plus variable cela implique en ce qui les concerne de faire des prélèvements rapprochés. Les concentrations en cadmium, tant dissous que particulaire, varient d'un ordre inférieur ce qui permet de réduire la fréquence du prélèvement tout en conservant une marge d'erreur sur les flux de cadmium comparable à celle obtenue sur ceux de MES (environ $8 \%$ ). II convient toutefois en cas de pollution subite ou de remobilisation importante soudaine (crues) d'augmenter cette fréquence.

\subsection{Site de prélèvement}

De 1990 à 1991 tous les prélèvements de MES et d'eau ont été effectués dans la zone du Lot aval à Clairac (fig. 1). A partir de 1992, en raison de la construction d'une écluse le site a du être déplacé à $8 \mathrm{~km}$ en amont, à Temple sur Lot (l'absence d'affluent entre ces deux points nous permet de considérer le bassin versant intéressé par les prélèvements comme identique). La prise de l'échantillonneur est située dans la veine principale du courant à une profon- 


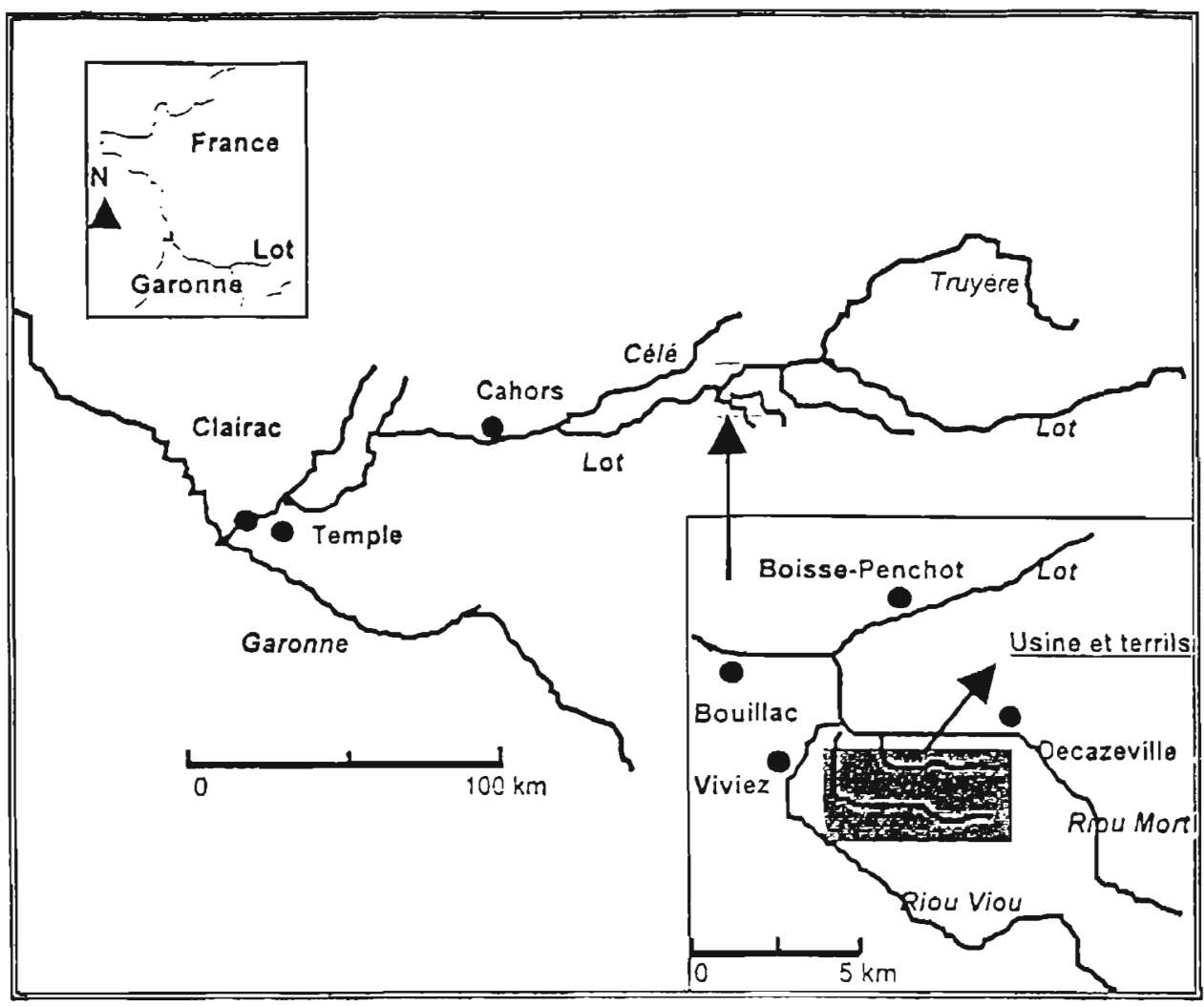

Fig. 1. - Carte du bassin du Lot et plan de localisation des sites d'échantillonnages.

Fig. 1. - Location map of the Lot River basin, showing place names and sample sites.

deur de 2 mètres et à environ 7 mètres de la berge. Pour vérifier l'homogénéité verticale et latérale de l'échantillonnage par le préleveur, deux cycles de mesures ont été effectués l'un en situation de crue et l'autre d'étiage sur la section de la rivière (fig. 2). L'étude du gradient des concentrations en MES a été faite sur des profils transversaux et verticaux, en surface, mi-profondeur et à 1 mètre du fond, en période d'étiage (24 points de mesure) et en cours de crue (26 points de mesure).
- En cours de crue, période de forts courants (de l'ordre de $1 \mathrm{~m} \cdot \mathrm{s}^{-1}$ ) et de fortes concentrations en MES on observe, sur la figure 2 , que le préleveur se situe bien dans la veine principale du courant, donc des flux principaux. La variation des MES à l'intérieur de cette section $\left(260 \pm 10 \mathrm{~g} \cdot \mathrm{m}^{-3}\right)$ est de l'ordre, en variation relative, de $3 \%$. Le flux de MES calculé à partir de la valeur donnée par le préleveur $\left(260 \mathrm{~g} \cdot \mathrm{m}^{-3}\right.$ ) est de 16130 t.j. $^{-1}$. L'évaluation faite en tenant compte du gradient des turbidités 


\section{Distribution en crue des courants et des MES - Lot à Temple}

18 Avril 1994 - Débit moyen $718 \mathrm{~m}_{3} \mathrm{~s}^{-1}$ - Concentration moyenne MES $260 \mathrm{~g} \cdot \mathrm{m}^{-3}$ - flux de MES $16000 \mathrm{i} . \mathrm{f}^{-1}$

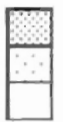

Concentration moy.MES $=265+$ ou $-10^{\prime} \mathrm{g} \cdot \mathrm{m}^{-3}$

Concentration moy. MES $=234+$ ou $-8 \mathrm{~g} \cdot \mathrm{m}^{3}$

Concentration moy.MES $204+$ ou- 20 g.m ${ }^{-3}$

\begin{tabular}{|l|l|}
\hline- & Point de mesure des MES \\
\hline 80 & Vitesse du courant $\mathrm{cm}^{-1}$
\end{tabular}

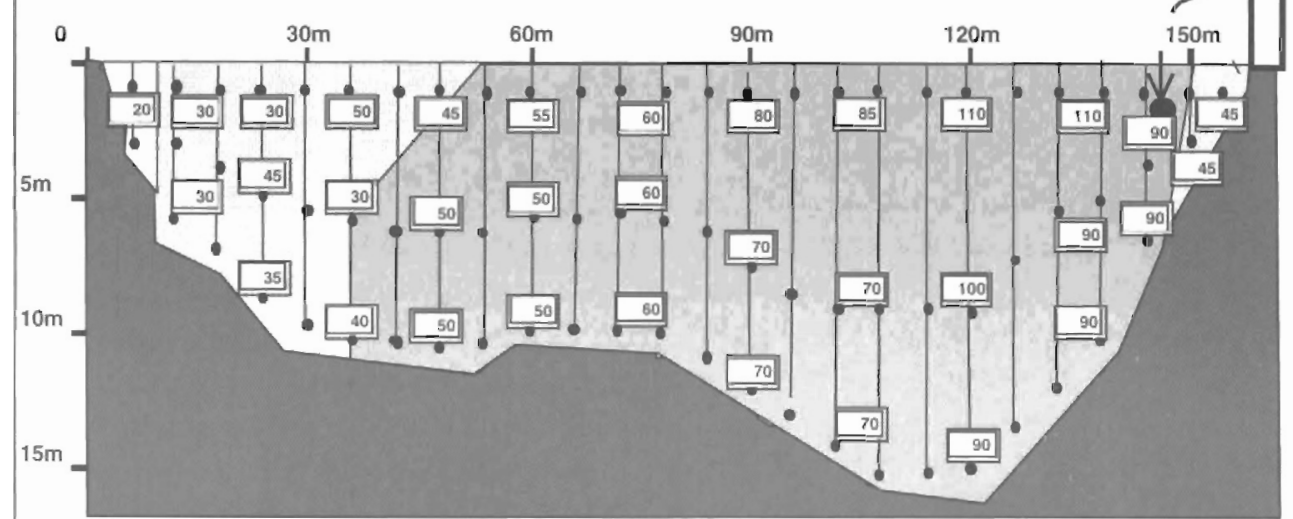

\section{Distribution en étiage des courants et des MES-Lot à Temple}

9 Juin 1994 - Débit moyen 37 m3.s $\mathrm{s}^{-1}$ - Concentration moyenne MES $17 \mathrm{~g} \cdot \mathrm{m}^{-3}$ - flux de MES $54 \mathrm{t} . \mathrm{j}^{-1}$
$\square$
Concentration moy. MES $=34 \mathrm{~g} \cdot \mathrm{m}^{-3}$
Concentration moy. IMES $14,5 \mathrm{~g} \cdot \mathrm{m}^{-3}$
- Point de mesure des MES
5 Vifesse du courant $\mathrm{cm} \cdot \mathrm{s}^{-1}$

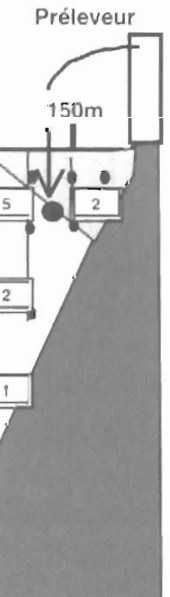

Fig. 2. - Distribution des MES et des courants, en crue et en étiage, sur une section du Lot a Temple.

Fig. 2. - TSS and stream distribution, during flood and low water, at Temple sur Lot. 
sur la section donne $15840 \mathrm{t.j}^{-1}$. L'écart relatif sur les flux (300 t.j $\left.j^{-1}\right)$ est ińférieur à $2 \%$.

- En étiage, les courants (quelques $\mathrm{cm} \cdot \mathrm{s}^{-1}$ ) et les MES (17 $\mathrm{g} \cdot \mathrm{m}^{-3}$ ) sont très faibles. La variation relative des concentrations en MES dans la section de prélèvement est de 1,2\%. Le flux de MES calculé à partir du préleveur (MES $=17 \mathrm{~g} \cdot \mathrm{m}^{-3}$ ) est de $54 \mathrm{t.j}{ }^{-1}$. Si l'on tient compte du gradient des

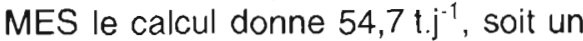
écart sur les flux de MES de 0,7.j $j^{-1}$ soit $1,3 \%$.

En résumé, en crue la mesure avec préleveur a révélé une surestimation des flux de MES d'environ $2 \%$, en étiage if $y$ a eu sous estimation de ces flux de l'ordre de 1,2\%. On a observé, par exemple : en situation normale de débit $\left(183 \mathrm{~m}^{3} . \mathrm{s}^{-1}\right)$ mais par forte pluie locale que le ruissellement crée momentanément au voisinage des berges une forte turbidité (entre 63 et $82 \mathrm{~g} \cdot \mathrm{m}^{-3}$, la concentration de MES relevé au préleveur est de $70,5 \mathrm{~g} \cdot \mathrm{m}^{-3}$ ) alors que les concentrations dans la partie centrale de la rivière sont de 24 à $35 \mathrm{~g} \cdot \mathrm{m}^{-3}$. L'erreur ici est importante (environ $50 \%$ ) mais l'impact sur les flux de MES est pondéré, du fait que le phénomène est relativement peu courant, et qu'il ne dure pas dans le temps; en effet au bout de quelques heures il y a homogénéisation des eaux et l'effet de berge disparaît.

\subsection{Les débits liquides}

L'étendue du bassin versant $\left(11840 \mathrm{~km}^{2}\right)$ et la diversité des types de climat déterminent une alimentation assez complexe (Massio, 1976) avec des précipitations méditerranéennes donnant de fortes pluies en automne sur le haut bassin du Lot, et des précipitations océaniques plus étalées et hivernales qui, intéressant la quasi totalité du bassin, provoquent les plus grandes crues en hiver (tableau 1). En année moyenne on peut observer classiquement un étiage d'été, mais les années sèches ont également un étiage d'hiver (janvier-février).

Les débits du Lot ont été mesurés, dans la zone aval, à la station de Villeneuve-sur-Lot située légèrement en

Tableau I. - Variations mensuelles du débit et des flux de MES en années sèche, humide et moyenne.

Table I. - Monthly variations of river discharge and TSS flux during dry, wet and mean year.

\begin{tabular}{|c|c|c|c|c|c|c|c|c|c|c|c|c|c|}
\hline & $\begin{array}{l}\text { Moy. } \\
\text { an }\end{array}$ & Janv. & Févr. & Mars & Avrit & Mar & Juin & Juill. & Aóut & Sept. & Oct. & Nov. & Déc. \\
\hline$\cdots \cdot$ & $\cdot$ & & & & & & . & & - & & & & - \\
\hline $\begin{array}{l}1991 \text { Année seche Q } \\
\text { Flux de MES M.t.an }\end{array}$ & $\begin{array}{c}107 \\
0.044\end{array}$ & $\begin{array}{c}74 \\
0.002\end{array}$ & $\begin{array}{c}144 \\
0,003\end{array}$ & $\begin{array}{c}212 \\
0.016\end{array}$ & $\begin{array}{c}176 \\
0.004\end{array}$ & $\begin{array}{c}146 \\
0.005\end{array}$ & $\begin{array}{c}60 \\
0.002 \\
-\end{array}$ & $\begin{array}{c}30 \\
0.001\end{array}$ & $\begin{array}{c}31 \\
0,001\end{array}$ & $\begin{array}{c}62 \\
0.002 \\
-\quad \cdots\end{array}$ & $\begin{array}{c}71 \\
0.002\end{array}$ & $\begin{array}{c}180 \\
0,004\end{array}$ & $\begin{array}{c}90 \\
0.002\end{array}$ \\
\hline $\begin{array}{l}1994 \text { Année humide } Q \\
\text { Flux de MES M.t.an }\end{array}$ & $\begin{array}{l}243 \\
0.77\end{array}$ & $\begin{array}{c}663 \\
0.333\end{array}$ & $\begin{array}{c}504 \\
0,156\end{array}$ & $\begin{array}{c}249 \\
0.020\end{array}$ & $\begin{array}{c}339 \\
0,085\end{array}$ & $\begin{array}{c}170 \\
0,010\end{array}$ & $\begin{array}{r}59 \\
0,003\end{array}$ & $\begin{array}{c}33 \\
0,002\end{array}$ & $\begin{array}{c}25 \\
0.001\end{array}$ & $\begin{array}{c}108 \\
0.009\end{array}$ & $\begin{array}{c}239 \\
0.046\end{array}$ & $\begin{array}{c}439 \\
0,101\end{array}$ & $\begin{array}{l}115 \\
0.059\end{array}$ \\
\hline $\begin{array}{l}\text { 1990-1995 O } \\
\text { Flux de MES M.t.a }\end{array}$ & $\begin{array}{c}157 \\
0.340\end{array}$ & $\begin{array}{c}215 \\
0.063\end{array}$ & $\begin{array}{c}251 \\
0.046\end{array}$ & $\begin{array}{c}198 \\
0,015\end{array}$ & $\begin{array}{c}183 \\
0.020\end{array}$ & $\begin{array}{l}128 \\
0.07\end{array}$ & $\begin{array}{c}125 \\
0.045\end{array}$ & $\begin{array}{c}93 \\
0,016\end{array}$ & $\begin{array}{c}37 \\
0.001\end{array}$ & $\begin{array}{c}69 \\
0.004\end{array}$ & $\begin{array}{c}1.35 \\
0.023\end{array}$ & $\begin{array}{c}253 \\
0.064\end{array}$ & $\begin{array}{c}210 \\
0.036\end{array}$ \\
\hline
\end{tabular}


amont $(10 \mathrm{~km})$ mais sans affluent intermédiaire par rapport au site d'échantillonnage. Les débits, fournis par le Service Hydrologíque et des Milieux Aquatiques de la Direction Régionale de l'Environnement de la région Midi-Pyrénées, sont les débits moyens journaliers. La précision sur les mesures est d'environ $5 \%$, elle est moins bonne pour les débits inférieurs à $40 \mathrm{~m}^{3} \mathrm{~s}^{-1}$ (de l'ordre de $20 \%$ ), mais cela ne concerne que les débits d'étiage sévère durant lesquels les flux de MES restent peu importants (Lapaquellerie et al., 1995).

\subsection{La détermination des MES}

La fréquence des prélèvements a d'abord été mensuelle, avec toutefois un suivi détaillé des principales crues (2 ou 3 prélèvements par jour de crue). Après les 2 premiers bilans réalisés en 1990 et 1991, il est apparu qu'une meilleure connaissance des flux de MES passait par un échantillonnage beaucoup plus serré des turbidités, notamment en période de crues. A partir de début 1992 la fréquence des prélèvements manuels devient journalière, puis dès 1993, l'échantillonnage devient automatique et journalier, intégrant un prélèvement toutes les 90 minutes. Le pas des prélèvements a été choisi suffisamment faible pour que les variations de débits liquides ou solides restent limitées dans cet intervalle de temps.

Un préleveur automatique (marque Bühler, type PB-MOS), fonctionne d'abord par refoulement de l'eau dans la crépine puis par aspiration. La cré- pine est située à environ $7 \mathrm{~m}$ de la berge et à $2 \mathrm{~m}$ de profondeur. Le prélèvement fournit un échantillon journalier moyen constitué de la sommation de 16 prélèvements élémentaires cumulés dans un flacon. Les volumes prélevés ont été déterminés de façon que le volume d'eau aspiré par la pompe fournisse un échantillon représentatif des MES de la rivière, en s'assurant de l'efficacité de la pompe. A partir de ces prélèvements les concentrations de matières en suspension (MES) ont été mesurées par filtration (filtre Whatman $G F / F$, porosité $0,45 \mu \mathrm{m}$ ) puis pesées sur des volumes de 0,5 à 1 litre d'eau. L'erreur relative de ces mesures de MES n'excède pas $1,5 \%$.

\subsection{Les concentrations en cadmium particulaire et dissous}

Des prélèvements spécifiques ont été réalisés systématiquement une fois par mois avec un échantillonnage spécial pour le suivi des crues, ce qui représente en année moyenne 40 échantillons.

- Cadmium particulaire: Le dosage du cadmium particulaire exige une quantité de matières de l'ordre du gramme, ce qui, compte tenu des turbidités, implique de traiter de gros volumes d'eau (de 500 à 1000 litres) mais présente aussi l'avantage de garantir une bonne représentativité de l'échantillon (prélèvement étalé sur 1 heure environ). Les MES sont séparées d'abord par centrifugation (séparateur Westfalia ayant un débit horaire de l'ordre de 135 litres); les parti- 
cules, soumises à une accélération de $12000 \mathrm{~g}$, sont récupérées avec un taux de $95 \%$, sans contamination métallique notable (Etcheber et Jouanneau, 1980). Les eaux à la sortie du séparateur sont ensuite filtrées, afin de recueillir les particules les plus fines, sur une batterie de filtres de $293 \mathrm{~mm}$ de diamètre (filtres Micropore polycarbonates de $0,45 \mu \mathrm{m}$ ) assurant un taux de récupération final de l'ordre de $98 \%$. L'utilisation successive de ces deux techniques permet ainsi de recueillir la quasi totalité des MES. Après récupération, les deux stocks sont séchés, mélangés de façon homogène et pesés puis soumis à l'analyse du cadmium.

- Cadmium dissous: Les prélèvements se font dans des flacons en polyéthylène de $100 \mathrm{~cm}^{3}$ décontaminés pendant 24 heures dans $\mathrm{HNO}_{3}$, Normapur $7 \mathrm{~N}$, à $50^{\circ} \mathrm{C}$, puis $\mathrm{HNO}_{3}$ Suprapur $1,5 \mathrm{~N}$ à $50^{\circ} \mathrm{C}$, puis rempli d'eau déionisée acidifiée à $\mathrm{HNO}_{3}$, avant d'être utilisés. Ce flacon est placé à l'intérieur d'un préleveur conçu en téflon qui permet d'effectuer le prélèvement liquide sans contact avec l'atmosphère. Afin d'éviter toute contamination, l'échantillon récupéré est immédiatement transféré sous la hotte à flux laminaire purifié, équipant le véhicule laboratoire de terrain, pour y être filtré (filtre Nuclépore polycarbonate de $0,45 \mu \mathrm{m})$. II est acidifie pour assurer la stabilisation chimique de la prise $(\mathrm{pH} 2)$ et conservé en enceinte réfrigérée jusqu'à l'analyse.

- Analyse chimique du cadmium particulaire et dissous: Pour la phase particulaire, la dissolution- extraction du métal fait appel à la digestion $\mathrm{HNO}_{3}$ et $\mathrm{HCL}$ en bombe téflon, selon la méthode de Loring, 1986.

Pour la phase dissoute, compte tenu des teneurs toujours assez élevées en cadmium, l'analyse se fait directement sur eau filtrée.

Dans tous les cas l'analyse élémentaire a été effectuée avec un spectromètre d'absorption atomique avec four. L'exactitude et la reproductibilité des mesures ont été testées à la faveur d'un exercice d'intercalibration de standards internationaux ICES (Loring, 1986) ce qui garantit la validité des résultats et la possibilité de comparaison dans le temps.

\section{RÉSULTATS}

\subsection{Hydrologie et turbidité}

Le nombre élevé d'observations permet de dégager des informations très significatives sur l'évolution hydrologique et sur les relations entre débits et charge de MES.

- Régime hydrologique. Si l'on compare (tableau 1) les débits annuels moyens mesurés à Villeneuvesur-Lot au cours de la période 1990-1995, avec la moyenne des débits annuels $\left(157 \mathrm{~m}^{3} \cdot \mathrm{s}^{-1}\right)$ de 1973 à 1995 on remarque que la période d'observation se révèle très représentative. Elle fait en effet apparaître, deux années de débits excédentaires (1992 et 1994), trois années de débits moyens (1990, 1993 et 1995) et une année déficitaire (1991). 
En fait, les débits relevés en 1990 et surtout en 1991, bien que faibles, ne traduisent pas totalement, à cause du soutient d'étiage dont bénéficie le Lot, la sévérité de la sécheresse relative à ces années là. II est préférable pour ces deux années de tenir compte du nombre de jours de crues où le débit a été supérieur à $400 \mathrm{~m}^{3} \cdot \mathrm{s}^{-1}$ (11 en 1990 et 2 en 1991). Celui-ci se révèle très faible comparé à la moyenne des 21 dernières années (tableau 2) qui est de 32 jours, et permet de considérer 1990 (avec un débit moyen de $126 \mathrm{~m}^{3} . \mathrm{s}^{-1}$ ) comme une année déficitaire sur le plan des flux de MES exportés.

- Relations débits-MES : Les résultats des mesures de la charge en MES en fonction des débits liquides journaliers pour l'ensemble des échantillons recueillis entre 1990 et 1995 ( $n=1927$ échantillons) sont reportés figure 3 .

Globalement, on observe une dispersion assez importante. Cependant plusieurs familles de points sont individualisés et font apparaître une rela- tion débit-MES significative pour une forte proportion des échantillions. En tenant compte de la pluviométrie locale au moment du prélèvement et des teneurs en carbone organique particulaire (COP) des échantillons, les ensembles suivants ont été distingués.

- Les mesures effectuées en période de fort ruissellement (précipitations supérieures à $20 \mathrm{~mm}$ par jour) sur le site même du prélèvement (figure 3 , étoiles noires) représentent 92 échantillons $(4,8 \%$ de la population) et ne correspondent pas à des situations de fronts de crue. Les importantes turbidités qui s'observent alors sont dues à des "bouffées" de MES liées au ruissellement des eaux de fortes pluies locales sur les berges proches du point de prélèvement. Les MES, surtout dans la zone proche des rives, peuvent être alors très abondantes, alors que la partie centrale de la rivière présente des concentrations de MES 2 ou 3 fois plus faibles (voir $\S \mathrm{II}-1)$. Ce phénomène peut durer quelques heures avant homogénéisation sur l'ensemble de la section. Ces

Tableau II. - Débits et flux de MES du Lot établis suivant deux méthodes flux mesurés au cours du suivi, et flux calculés à partir de la relation (1).

Table II. - Annual volume discharge and TSS flux calculated using two different methods.

\begin{tabular}{|c|c|c|c|c|c|c|c|}
\hline Années & $\begin{array}{r}1973- \\
1995\end{array}$ & 1990 & 1991 & 1992 & 1993 & $\vdots 1994$ & : 1995 \\
\hline Débits annuels moyens $\mathrm{m}^{3} \cdot \mathrm{s}^{-1}$ & 157 & 126 & 107 & 198 & 128 & 243 & 141 \\
\hline Nombre de jours (débit $>400 \mathrm{~m}^{3} \cdot \mathrm{s}^{-1}$ ) & 32 & 11 & 2 & 40 & 14 & 85 & 32 \\
\hline Flux annuel de MES mesuré (suivi) Mt.an ${ }^{-1}$ ) & & 0,045 & 0,044 & 0,670 & 0,280 & 0,842 & 0,216 \\
\hline Flux annuel de MES calcu & & 0,161 & 0,109 & 0,542 & 0,260 & 0,822 & 0,220 \\
\hline
\end{tabular}


MES $\mathrm{g} \cdot \mathrm{m}^{-3}$

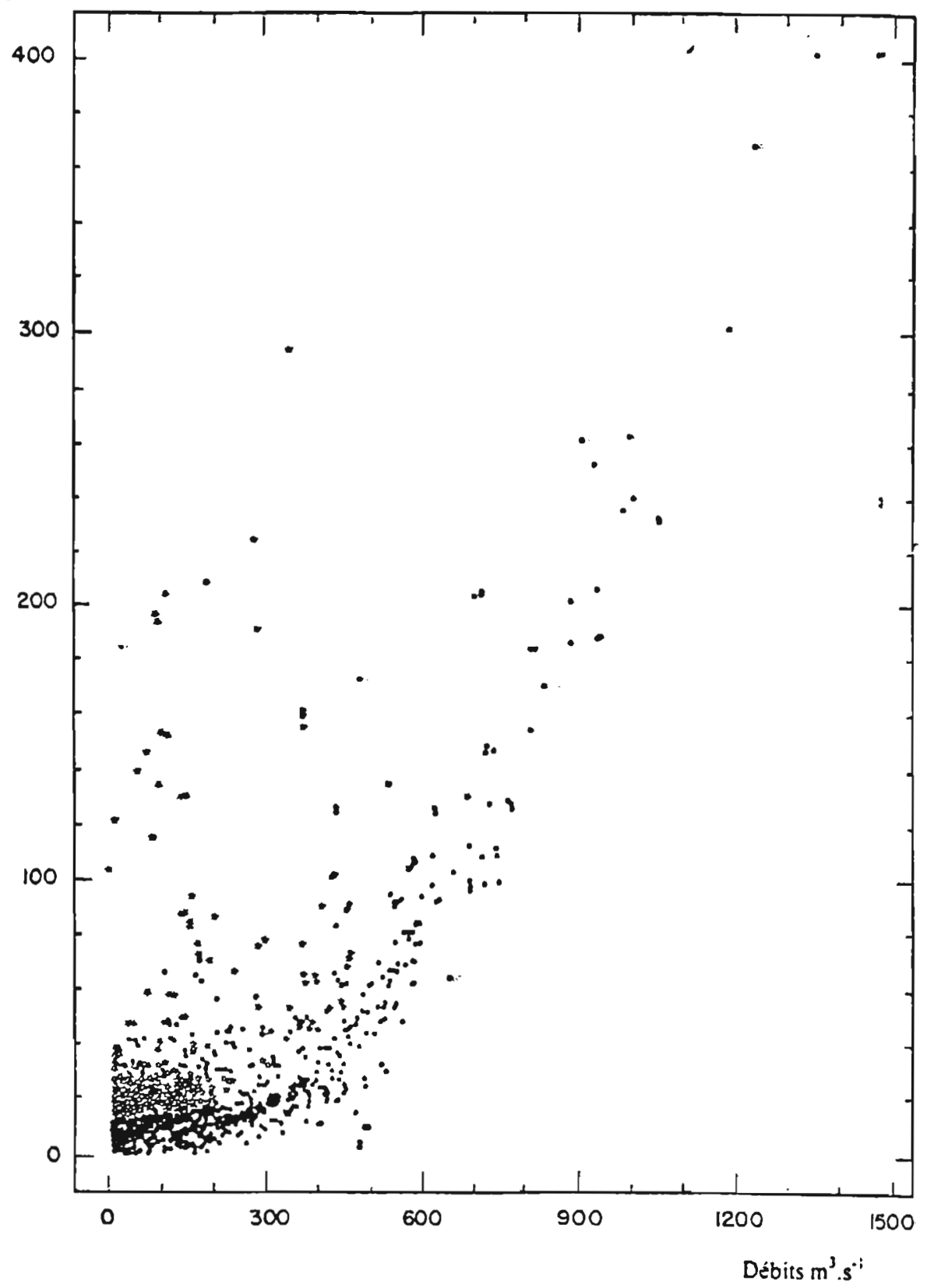

Fig. 3. - Représentations des concentrations de MES (1927 mesures) en fonction du débit du Lot (1990-1995). Les étoiles pleines $(4 \%)$ représentent les échantillons prélevés en période de fort ruissellement; les étoiles claires $(22 \%)$ ceux correspondant à de fortes concentrations en COP; les points noirs $(73 \%)$ représentent les échantillons prélevés au cours de conditions plus "normales".

Fig. 3. - Plot of 1927 daily measurerments of TSS vs. corresponding volumetric discharge in the Lot River (1990-1995). Solid stars (4\%) represent measurements collecled during periods of hight surface runoff; open stars $(22 \%)$ represent those with hight organic content; solids dots $(73 \%)$ represent those collected during more "normal" conditions. 
effets de ruissellement locaux sont amplifiés par le fait que les sols proches de la vallée du Lot, à forte vocation agricole, se caractérisent par une érodabilité élevée (Parde, 1963; Probst, 1983; Etchanchu et Probst, 1986).

\section{- Les échantillons recueillis lors} des périodes d'étiage (figure 3 , étoiles blanches) où les débits sont inférieurs à $40 \mathrm{~m}^{3} . \mathrm{s}^{-1}$ et où les concentrations en carbone organique particulaire sont supérieures à $7 \%$ et peuvent atteindre $28 \%$ en fin de période estivale. Pour ces 423 échantillons (soit $22 \%$ de la population) les concentrations en MES sont relativement élevées. Ces mesures correspondent à des prélèvements de printemps et d'été, périodes sujettes à de forts développements végétaux d'origine autochtone (floraisons planctoniques, lentilles d'eau). Cette prolifération qui s'accompagne de l'eutrophisation des fonds bien caractéristique de la vallée du Lot (Agence de l'Eau Adour-Garonne, 1993) semble être le résultat de l'action humaine au cours des dernières décennies avec une agriculture intensive et la création de nombreuses retenues (62) sur l'ensemble du Lot provoquant la modification de la qualité physico-chimique des eaux (Bengen et al., 1992; Belaud, 1994).

\section{- Enfin la population restante} (figure 3 , points noirs) qui représente la majorité des cas (1 472 échantillons, $73 \%$ de la population initiale) correspond aux points où la concentration en MES varie avec le débit. La relation (1) entre concentration ( $C_{\text {MES }}$ ) de MES et le débit ( $\mathbf{Q}$ ) est exprimée suivant l'expression générale recommandée par Meybeck et al., 1994 :
(1)

$$
\left(C_{\text {MES }}\right)=a \cdot Q_{(\text {Débits) }}{ }^{b}
$$

Dont les constantes calculées sont : $a=0,0004$ et $b=1,92$.

Le coefficient de corrélation entre MES et débits est $r=0,84$.

Commentaires : La caractérisation des systèmes fluviaux a été fréquement recherchée sur la base des relations débits-MES dans les eaux. Souvent la corrélation obtenue est médiocre et il est admis (UNESCO, 1988) que cela tient à la fois à la complexité des processus contrôlant la charge solide en suspension et aux incertitudes des mesures (difficultés d'échantillonnage lors des crues, stations de prélèvements inadéquates, représentativité de l'échantillonnage, etc.). La représentativité de l'échantillonnage peut être ici considérée comme très bonne et grâce à cela la relation débit-MES représentative de $73 \%$ des points a été obtenue avec une probabilité de l'ordre de $95 \%$.

De plus les relations débits-MES ont été contrôlées au cours de divers types de crues du Lot : crues de printemps (juin 1992, avril-mai 1993, avrilmai 1994) et crues d'automne-hiver (octobre-novembre 1992, décembre 1993, janvier et février 1994) mais aussi en période d'étiage sévère. Un exemple est fourni figure 4 représentant le suivi journalier des MES en fonction des débits. 
Crue du Lot (Temple- Sept_-Oct. 1992)

Coupbes de tendance (regr. polyn.) du Cd dissous et particulairo compares au dibit ot aux MES

900

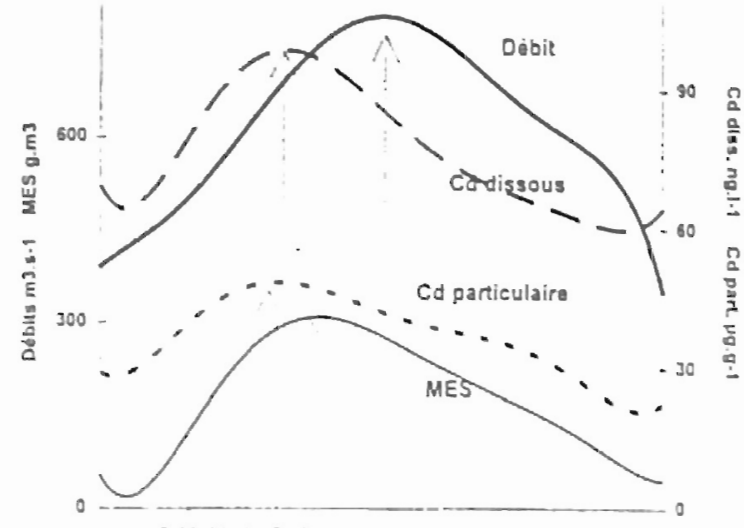

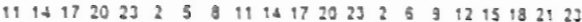

$\mathrm{HHHHHHHHHHHHHHHHHHH}$

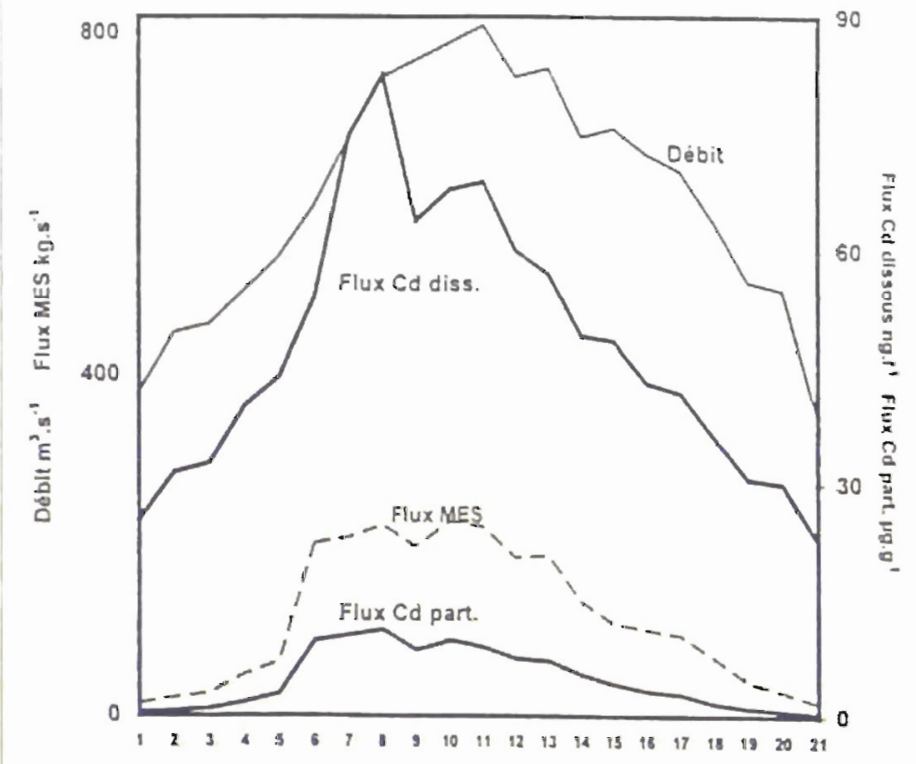

Fig. 4. - Variation horaire des concentrations et des flux de Cd en fonction des débits et de la charge en MES au cours de la crue du Lot du 30 septembre 11 heures au 1 octobre 23 heures (1992).

Fig. 4. - Plot of concentations and fluxes of Cd with volume discharge and TSS of Lot River during the floods of 30 September to 1 October. 


\subsection{Les flux de M.E.S.}

Les méthodes de calcul des flux de MES et de polluants associés ont fait l'objet d'un grand nombre de travaux (Balland, 1983 et 1984; Walling, 1977; Walling and Webb, 1981; I.M.O. et al., 1987; Etchanchu, 1988); on citera plus particulièrement ceux de Rakoczi (1977), de Meade and Parker (1985) et de Meade and Stevens (1990). La méthode dite stochastique ou des flux mesurés, préconisée par Meybeck (1992) a été utilisée ici pour quantifier les flux de MES. Dans cette méthode on considère que la concentration en MES mesurée au cours du prélèvement est représentative des concentrations relatives à la tranche de temps séparant deux prélèvements. Ce procédé est bien adapté aux données obtenues avec un préleveur automatique; il permet de calculer les flux journaliers à partir de la concentration moyenne en MES ( $\mathbf{C}_{\text {MES }}$ ) de 16 prélèvements quotidiens et du débit moyen journalier (Q). Le flux annuel de MES ( $\boldsymbol{F}_{\text {MES }}$ ) est la sommation sur l'année du produit suivant :

$$
\text { (2) } \quad F_{\text {MES }}=\sum_{1}^{365} Q \cdot C_{\text {MES }}
$$

Compte tenu de l'erreur de jaugeage portant sur les débits, de l'incertitude sur l'évaluation des MES et de l'erreur de calcul, on peut estimer qu'en valeur relative l'erreur totale sur les flux de MES est d'environ $15 \%$. Bien entendu, le calcul ne prend pas en compte le transport des sédiments sur le fond qui, eux, suivent un autre processus de transport que les MES.
Etant donné le grand nombre de barrages échelonnés le long du Lot, on peut considérer que les transports par le fond sont extrêmement réduits.

A titre de comparaison et afin de tester une méthode beaucoup plus simple qui pourrait être éventuellement utilisée dans l'avenir, nous avons calculé les flux de MES en appliquant l'équation (1). Dans le cas présent la relation établie n'est représentative que de $73 \%$ des échantilIons. Le calcul en terme de flux établit en tenant compte de l'échantillonnage total montre que le flux de MES est représenté à $86 \%$ et celui du cadmium à $91 \%$. L'écart important sur le flux de MES est lié au fait que les apports d'origine locale sont importants sur le plan des MES et négligeables sur celui du cadmium et, inversement le flux de MES d'origine organique est faible mais reste déterminant car riche en cadmium (chélation des molécules métalliques, concentration par les organismes, etc.), on peut l'estimer comme responsable des écarts du flux de cadmium (9\%). L'incertitude relative aux flux de MES avoisine les 30\% (14\% par rapport à la méthode précédente et $15 \%$ pour l'erreur liée aux mesures, ajustement de la régression, etc.), à comparer avec la méthode précédente dont la précision est de l'ordre de $15 \%$.

Les résultats obtenus par les deux méthodes sont reportés dans le tableau 2. Pour les années de faibles débits et de soutient d'étiage par lâchers de barrage du haut bassin versant, comme en 1990 et 1991, ce 
décalage est important entre les deux méthodes. Dans ces conditions la relation débit-concentration de MES ne dépend pas uniquement de la dynamique de la rivière mais aussi des phénomènes connexes (développements planctoniques, sédimentation dans les nombreuses retenues). Par contre, pour les autres années, lorsque le flux de MES est de l'ordre ou supérieur à $0,2 \mathrm{Mt}^{-a^{-1}}$ les résultats sont du même ordre et les écarts relatifs ne dépassent pas $20 \%$.

Commentaires : L'expérience menée de 1990 à 1995 a ainsi permis de dégager une relation débit-concentration de MES significative car elle intégre un intervalle de temps avec saisie de conditions hydrologiques extrêmes où ont alterné des périodes de grandes crues et des passages en étiage très sévères agrémentés de fort soutien d'étiage.

Pour une évaluation rapide mais moins précise des flux annuels de MES dans le Lot on peut ainsi faire appel à la relation (2). Toutefois ce calcul n'est basé que sur $73 \%$ des échantillons mais ceux-ci représentent en flux de MES $86 \%$ du flux annuel total. L'incertitude est plus grande, mais la méthode présente l'avantage de pouvoir fournir, à partir des seuls débits, une estimation rapide des flux exportés sur 1 an. Une estimation ponctuelle sur une période plus courte à l'échelle du mois est aléatoire. On remarque que (tableau 1) les principales exportations de MES se produisent en janvier et novembre ce qui correspond aux plus forts débits moyens de la période 1990-1995.
En résumé : dans le cas du Lot en particulier, l'obtention d'un bilan des flux de MES fiable, à l'échelle de l'année, passe obligatoirement par une fréquence d'échantillonnage élevée. Le bilan des flux ainsi obtenu est suffisamment fiable pour servir de base de calcul des flux polluants émis sous forme particulaire par le Lot et exportés ensuite vers la Garonne.

\subsection{Les flux de cadmium}

Les principales difficultés dans le suivi de l'évolution des flux de cadmium à long terme consistent :

- à éviter pour le cadmium dissous en particulier la contamination des échantillons lors des prélèvements. Cette difficulté a pu être surmontée grâce à l'utilisation d'un protocole méthodologique rigoureux;

- à assurer la continuité de la méthodologie et cela en dépit des changements imposés au système durant le suivi (exemple de la construction d'une écluse ayant obligé à déplacer la station de mesure des MES vers l'amont) et les changements résultant de l'amélioration des techniques disponibles (exemple: préleveur automatique);

- à vérifier constamment la répétabilité des analyses chimiques dans le temps (standards témoins, intercalibration).

- Flux de cadmium dissous (tableau 3) : les flux de cadmium dissous $\left(F_{\text {Cdd }}\right)$ sont établis à partir du produit de la concentration moyenne pondérée par le volume écoulé. 
Tableau III. - Flux annuels de Cd dissous expulsés par le Lot (1990-1995).

Table III. - Annual flux of dissolved Cd for the Lot River (1990-1995).

\begin{tabular}{|c|c|c|c|c|c|c|}
\hline Années & 1990 & 1991 & 1992 & 1993 & 1994 & 1995 \\
\hline Nombre d'analyses & 15 & 20 & 20 & 18 & 28 & 30 \\
\hline Concentrations moyennes de $\mathrm{Cd}$ en $n g . \mathrm{I}^{-1}$ & 295 & 261 & 112 & 121 & 119 & 67 \\
\hline Flux annuel de Cd dis & $1,2 \pm 0,2$ & &, $7 \pm 0$ &, $5 \pm 0$ & $0,9 \pm$ & \\
\hline
\end{tabular}

$$
F_{C d d}=\bar{Q} \cdot\left(\sum_{i} Q_{i} \cdot C_{i}\right)
$$

$\bar{Q}=$ débit moyen, $Q_{i}=$ débit instantané correspondant à l'échantillon instantané, $C_{i}=$ concentration instantanée.

On observe généralement que l'ordre de grandeur des flux dissous reste relativement constant depuis 1990. L'arrivée subite d'un flux contaminant peut survenir comme par exemple lors d'une remobilisation des sédiments contaminés dans le lit du Lot au cours d'une crue. II y a assez bonne concordance (fig. 4), mais avec un décalage dans le temps de l'ordre de 12 heures, entre concentration de cadmium dissous et débit (voir courbe de tendance obtenue par régression polynomiale) et flux de cadmium dissous et débit.

- Flux de cadmium particulaire: Le mode de calcul du flux de cadmium particulaire $\left(\boldsymbol{F}_{\mathrm{Cdp}}\right)$ en fonction de la concentration de cadmium $\left(F_{\text {Cdp }}\right)$ est celui recommandé par Meybeck et al., 1994 dans le cas où :

- les flux de métal particulaire sont dépendants de la variation dans le temps des concentrations en MES (fig. 4);
- les variations des teneurs en métal particulaire sont peu rapides dans le temps et autorisent un nombre pas trop élevé d'analyses;

- il est possible d'obtenir une bonne connaissance des flux de MES $\left(\mathrm{F}_{\text {MES }}\right)$.

Ces conditions étant celles caractérisant le cas du Lot, les flux de cadmium particulaire (tableau 4) ont été estimés à partir de la relation suivante :

$$
\mathrm{F}_{\mathrm{Cdp}}=\sum \mathrm{F}_{\mathrm{MES}} \cdot \mathrm{C}_{\mathrm{Cdp}}
$$

Au cours de la crue d'octobre 1992 à Temple, on observe une bonne concordance entre concentration de cadmium particulaire et de MES (avec un retard d'environ 8 heures pour les MES) et aussi avec les flux de cadmium et de MES (fig. 4).

- Flux de cadmium total: Ces flux (sommes des flux de cadmium dissous et particulaire) sont reportés tableau 5.

La relation entre débit annuel moyen et flux de cadmium est particulièrement évidente. Aux années de sécheresse (1990 et 1991) correspondent des flux de cadmium peu importants et durant les années plus 
Tableau IV. - Flux annuels de Cd particulaire expulsés par le Lot (1990-1995).

Table IV. - Annual flux of particulate Cd for the Lot River (1990-1995).

\section{Années \\ Nombre d'analyses}

Concentrations moyennes de Cd en $\mu \mathrm{g} \cdot \mathrm{g}^{-1}$

Flux annuel de Cd particulaire $\tan ^{-1}$

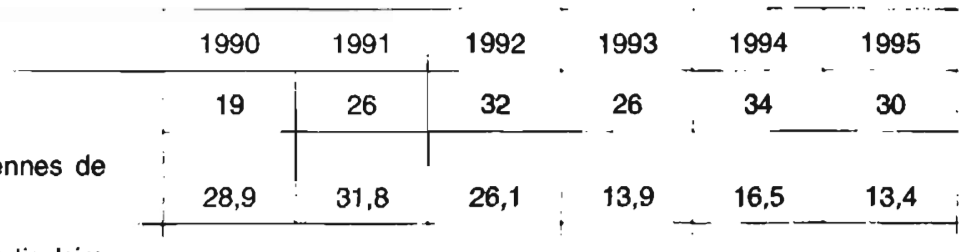

$1,3 \pm 0,3: 1,4+0,2: 17,5 \pm 2,7: 3,9 \pm 1,1 \cdot 13,9 \pm 3,1: 2,9+0,5$

Tableau V. - Flux annuels de Cd total expulsés par le Lot (1990-1995).

Table V. - Annual flux of total Cd for the Lot River (1990-1995).

\begin{tabular}{|c|c|c|c|c|c|c|}
\hline ot & 1990 & 1991 & 1992 & 1993 & 1994 & 95 \\
\hline 1 & $2,5=$ & $2,3 \pm$ & $18,2 \pm 2,9$ & 4,4 上 1,3 & $14,8 \pm 3,2$ & $3,2 \pm 0,6$ \\
\hline
\end{tabular}

humides (1992 et 1994) on observe des flux de cadmium beaucoup plus élevés. Ainsi apparaît-il qu'en dépit de l'isolement de la source polluante initiale, des flux très importants de cadmium peuvent être encore exportés en Garonne et au-delà vers l'estuaire de la Gironde.

Le suivi des flux polluants de cadmium exportés vers la Garonne permet de prévoir l'évolution du stock de cadmium piégé dans le lit de la rivière. Ce stock a été évalué à 191 t en 1990 (Lapaquellerie et al, 1995) et, compte tenu des expulsions depuis cette époque, il peut encore être estimé à $150 \mathrm{t}$ en 1996. Ce suivi de l'évolution permet de juger de l'amélioration de l'ensemble du bassin et de la réduction progressive de son état de pollution. On peut ainsi (tableaux 4 et 5) suivre l'évolution des concentrations pondérées des débits liquides et des débits solides entre 1990 et 1995 (on s'affranchit des conditions hydrologiques responsables de fortes variations des flux liquides et de flux de MES) au cours du temps.

Sur la période observée il ressort que le cadmium dissous marque une diminution brutale des concentrations entre 1990 et 1992 suivie d'une relative constance des valeurs avec toutefois une tendance à la baisse à partir de 1995. Les concentrations en cadmium particulaire diminuent constamment (tableau 4) de 1990 à 1995 avec toutefois une légère remontée en 1994 (année où les fortes crues ont remobilisé des sédiments d'autant plus anciens, plus riches en cadmium particulaire, que la crue est puissante, entraînant une remontée annuelle des concentrations).

Même si les flux polluants restent encore importants lors des années humides il semble que l'on s'achemine lentement vers une diminution globale de ces flux. 


\section{CONCLUSION}

La méthodologie retenue pour le suivi des flux de MES sur un long terme a fait l'objet d'une reconnaissance préalable entre 1986 et 1989. Cette étude préliminaire a permis de définir une stratégie globale bien adaptée au régime hydrologique de la rivière régulée par un nombre important de barrages et de retenues mais présentant des périodes à forts débits et des variations de la nature des MES dues principalement aux fortes teneurs en COP durant les étiages. II est alors apparu qu'une connaissance correcte du débit solide impliquait l'élaboration progressive d'un suivi systématique des concentrations en MES. L'automatisation des prélèvements a eu pour conséquence une fréquence d'échantillonnage nécessaire pour une meilleure estimation des charges solides pour les années humides mais qui pourrait être jugée redondante en période d'étiage.

L'étude des métaux dissous a impliqué de travailler en conditions ultra-propres (création d'un matériel de récupération spécifique, décontamination du matériel de prélèvement, laboratoire mobile propre équipé d'une hotte à flux laminaire, etc.). De même la récupération de quantités importantes de MES demande un matériel de terrain lourd et délicat.

Le choix du mode de calcul des flux de cadmium particulaire et dissous découle directement de la stratégie d'échantillonnage. Le nombre important de mesures réalisées à per- mis de faire ressortir une relation concentrations de MES-débits liquides significative. Cependant cette relation n'est pas suffisamment représentative de l'ensemble des échantillons pour pouvoir se passer des mesures de MES pour le calcul des flux de métaux (crues, ruissellements, bloom planctonique). Cela justifie un suivi des flux polluants impliquant l'utilisation d'une méthodologie assez lourde permettant en particulier de suivre en détail les exportations majeures liées aux crues.

\section{REMERCIEMENTS}

Ce travail a été rendu possible grâce au support accordé par le C.N.R.S. dans le cadre de I'URA 197 et au soutien financier accordé par le G.I.S. ECOBAG.

Nous tenons à remercier $M$. Marasse (EDF Cajarc), M. Mas (EDF Temple) et M. Tremblin (EDF Castelnau-Lassouts) ainsi que leurs équipes pour leur disponibilité et toute l'aide qu'ils ont bien voulu nous dispenser au cours de ce travail.

\section{RÉFÉRENCES BIBLIOGRAPHIQUES}

Agence de l'Eau Adour-Garonne, 1993. L'eutrophisation étouffe nos rivières, Rapport, $10 \mathrm{p}$.

Balland P., 1983. Méthodologie de quantification des flux de nutriements en rivières. Proc. Of Hambourg Symp., A.I.H.S., Publ. 141, 333-345.

Balland $P ., 1984$. Utilisation de la relation Concentration-Débit pour le calcul des flux de matières en rivières. Physio Geo, 9, 5-13. 
Belaud A., 1994. Rôle et intérêt des bras morts de la Garonne. Rev. Agence de l'Eau Adour-Garonne, 60, 21-25.

Bengen D., Lim P. et Belaud A., 1992. Qualité des eaux de trois bras morts de la Garonne : variabilité spatio-temporelle. Rev. Sci. De l'Eau, 5, 131-136.

Boisseau P., 1987. Pollution accidentelle du Lot: Le flux polluant Adour-Garonne. Rev. Agence de l'Eau AdourGaronne, Vol. 34, 7-9.

Boutier B., 1981. Synthèse des résultats de la surveillance des micropolluants dans la matière vivante. Ministère de l'Environnement, Bulletin du Réseau National d'Observation, 17, 115-174.

Boutier B., 1990. Le cadmium : Propriétés, utilisation, conséquences sur l'environnement marin. Rapp. interne Ifremer, $24 \mathrm{p}$.

Etchanchu D., 1988. Géochimie des eaux du bassin de la Garonne, transfert de matières particulaires et dissoutes vers l'Océan Atlantique. Thèse d'Univ., Université P. Sabatier, Toulouse, $178 \mathrm{p}$.

Etchanchu D. et Probst J.L., 1986. Erosion et transport des matières en suspension dans un bassin versant en région agricole. Méthode de la mesure du ruissellement superficiel, de sa charge et des deux composantes du transport solide dans un cours d'eau. C.R. Acad. Sci. Paris, t. 302, Série II, 17, 1063-1068.

Etcheber H. and Jouanneau J.M., 1980. Comparison of the different methods for the recovery of suspended matter from estuarine waters deposition, filtration, and centrifugation; consequences for the determination of some heavy metals. Estuar. Coast. Mar. Sc.; Londres, II, 701-707.

I.M.O. / F.A.O. / U.N.E.S.C.O. / W.M.O. / I.A.E.A. / U.N. / U.N.E.P., 1987. Land sea boundary flux of polluants. Final report of working group 22.
Jouanneau J.M., 1983. Matières en suspension et oligo-éléments métalliques dans le systeme estuarien girondin: comportement et flux, Mem. Inst. Géol. Bassin d'Aquitaine Bordeaux, 16, $306 \mathrm{p}$.

Jouanneau J.M., Boutier B., Chiffoleau J.F., Latouche C. and Philips I., 1990. Cadmium in the Gironde Fluvioestuarine System: Behaviour and Flow. The Science of the Total Environment, $97 / 98$, p. $465-469$.

Lapaquellerie Y., Jouanneau J.M., Maillet $\mathrm{N}$. et Latouche C., 1995. Pollution en cadmium des sédiments du Lot (France) et calcul du stock de polluant. Environmental Technology, Vol. 16, pp. 1145-1154.

Latouche C., 1970. Nature et évolution des cortèges argileux dans le systeme Garonne-Dordogne-Gironde. C.R. Acad. Sci. Paris 271, pp. 2080-2083.

Latouche C., Jouanneau J.M. et Philips I., 1989. Etude de la distribution du cadmium dans les bassins versants de la Dordogne de la Garonne et du Lot. Rapport Agence de l'Eau Adour-Garonne, $\mathrm{pp} .1-71$.

Loring D.H., 1986. ICES Intercalibration for Trace Metals in Marine Sediment, Rap. Deart. Of Fisheries and Ocean Atlantic Oceanography, Dortmouth, N.S. B2Y 4A2, Canada, pp. 1-64.

Massio J.C., 1976. Facteurs d'évolution des matières en suspension minérales et organiques dans les eaux du Lot. Thèse, Univ. P. Sabatier, Toulouse, $135 \mathrm{p}$.

Meade H. And Parker R.S., 1985. Sediment in river of the United States. Nat. Water Summary U.S. Geol. Surv. Water-Supply Paper $n^{\circ} 2275$, pp. 49-60.

Meade H. and Stevens H.H., 1990. Strategies and equipment for sampling suspended sediment and associated toxic chemicals inlarge rivers - with 
emphasis on the Mississipi river. Sci. Tot. Envir. 97-98, pp. 125-135.

Meybeck M., 1992. Etablissement des flux polluants. Rapport final, Agence de l'Eau Adour-Garonne.

Meybeck M., Pasco A. et Ragu A., 1994. Etablissement des flux polluants dans les rivières pourquoi, comment et à quel prix? Rencontres de l'Agence Régionale pour l'Environnement. Provence-Alpes-Côte d'Azur. Colloque scientifique sur les charges polluantes véhiculées par les fleuves et les rivières en Méditerranée? Collection 1991-1992-1993.

Parde M., 1963. Quelques notions nouvelles ou améliorées sur l'érosion et les transports solides. C.R. Assemblée de Berkeley, Publ. 65, pp. 166-176.

Probst J.L., 1983. Hydrologie du Bassin de la Garonne. Modèles de mélange, bilan de l'érosion. Exportation des phosphates et des nitrates, Thèse Univ. P. Sabatier, Toulouse, $167 \mathrm{p}$.
Rakoczi L., 1977. The significance of infrequent, hight suspended sediment concentrations in the estimation of annual sediment. Int. Ass. Hydrol. Sci. Publ., 122 p.

Roux M. et Simonet F., 1987. Pollution accidentelle du Lot. Flux polluant. Rev. de Agence de l'Eau Adour-Garonne, N.34/Hiver 1987, p. 7-9.

UNESCO, 1988. River inputs to Ocean Systems: Status and recomendations for research, Tech. Papers in Marine Science, 55, $25 \mathrm{p}$.

Walling D.E., 1977. Limitation of the rating curve technique for estimating suspended sediment loads, with particular reference to British rivers. Proc. of Paris Symp. A.I.H.S., Publ. 122, 34-48.

Walling D.E. and Webb B.W., 1981. The reliability of suspended sediments load data. Proc. of Florence Symp., A.I.H.S. Publ. 137, pp. 409-419. 